\title{
Kinking of the Outflow Graft, Consequent Ventricular Tachycardia, and the Need for Reoperation in a Patient with Left Ventricular Assist Device
}

\author{
Dusko Terzic, MD, Emilija Nestorovic, MD, Svetozar Putnik, MD, PhD, Dejan Markovic, MD, \\ Miljko Ristic, $\mathrm{MD}, \mathrm{PhD}$ \\ Department for Heart Transplant, LVAD and ECMO, Clinic for Cardiac Surgery, Clinical Center of Serbia, Belgrade, Serbia
}

\section{ABSTRACT}

Background: Left ventricular assist devices (LVAD) have become a lifesaving solution for patients awaiting heart transplantation as well as an option to support the failing hearts of non-transplant candidates as a lifelong, or destination therapy (DT). Improvements in LVAD design have enabled greater durability and broader patient applicability, but not without complications. Ventricular arrhythmias in LVAD patients were considered benign in the early days of LVADs, but today are increasingly recognized for their harmful impact on morbidity and quality of life.

Case presentation: We describe a 53-year-old male who underwent HeartWare left ventricular assist device (HVAD) implantation. During the postoperative period, the patient experienced ventricular tachycardia (VT) during a coughing episode, later found on CT to be due to significant angulation of the outflow graft. Following reoperation to shorten the outflow graft, the patient returned to hemodynamic stability, without VT or other arrhythmias.

Conclusions: Innovative strategies in VT prevention and improved clinical outcomes in LVAD patients may be the result of better understanding of characteristics that predispose these patients to VT. This case report showed that an excessively long outflow graft with considerable kinking created significant VT, but reoperation to correct the length of the graft mitigated further VT complications.

\section{CASE REPORT}

A 53-year-old male, body surface area $1.92 \mathrm{~m} 2$, was implanted as a BTT with a HeartWare LVAD (HVAD; Heart Ware, Framingham, MA, USA) due to end-stage ischemic heart failure. He was admitted to our hospital with deteriorating heart function despite maximal medical treatment. Medical history included insulin-dependent diabetes mellitus, hyperlipidemia, chronic renal insufficiency, and cerebrovascular disease. There was no history of heart rhythm disturbances. The patient initially had an uneventful postoperative period. However, after extubation, on postoperative day 3 the patient began to experience new onset VT while sitting upright and coughing; a time when venous blood flow

\footnotetext{
Received fune 6, 2017; accepted fune 16, 2017.

Correspondence: Duško Terzic, Clinic for Cardiac Surgery, Clinical Center of Serbia, Belgrade, Serbia, Dr: Koste Todorovica 8, 11000 Belgrade; +381668301966 (e-mail: terzic.dusko@gmail.com).
}

to the heart is normally reduced. Despite administration of anti-arrhythmic medications, he continued to experience hemodynamic instability. A 24-hour Holter ECG monitor showed sinus rhythm the majority of the time, with an average heart rate (HR) of $85-90$ beats per min (bpm). During sleep, the HR stayed a minimum of $72 \mathrm{bpm}$, with a maximum of $106 \mathrm{bpm}$ during moderate activity (lying and half-sitting position). Other findings showed premature ventricular extra systole, in total ventricular extra systole below 1000/24.

Having excluded an electrophysiological cause, an MSCT of the chest was performed to detect possible mechanical causes. This scan revealed an unclear proximal portion of the outflow graft due to artifact. The distal half of the graft to the aorta revealed tortuous flow with significant angulation (Figure 1). Based on clinical parameters and MSCT findings, the decision was made to return the patient to the operating room to resolve outflow graft kinking.

The patient underwent reoperation on postoperative day 12. The patient remained hemodynamically stable. Dual inotropic support (dobutamine and norepinephrine) was initiated proactively in preparation for short pump stop during outflow graft repair. Full dose heparin was administered in case extracorporeal circulation was needed, which wasn't the case. After resternotomy and lysis of adhesions, it was confirmed the outflow graft was too long, resulting in kinking. Hemodynamic parameters were satisfactory after dual inotropic support and volume resuscitation, so the surgeon estimated it was safe to stop the HVAD for 8 minutes after outflow graft clamping. There were no hemodynamic repercussions. The surgeon shortened the outflow graft by $6 \mathrm{~cm}$ without incident. The graft was reaerated, declamped, and

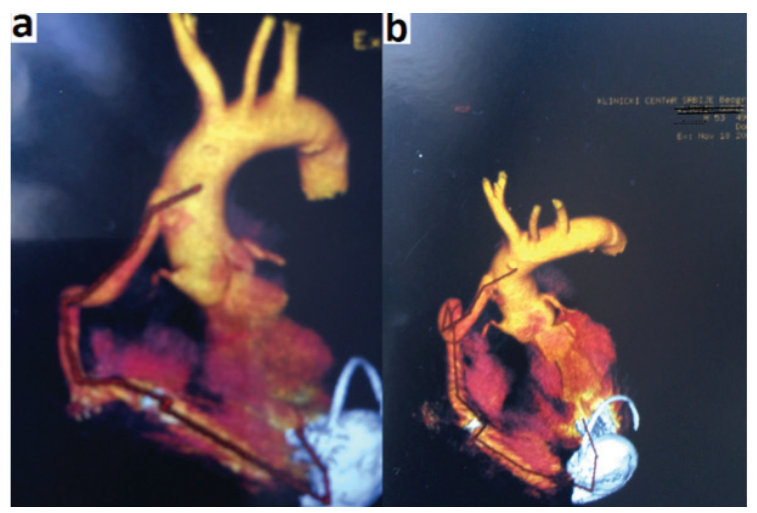

Figure 1. CT scan before correction (A). The distal half of the graft to the aorta tortuous flow with significant angulation (B). 


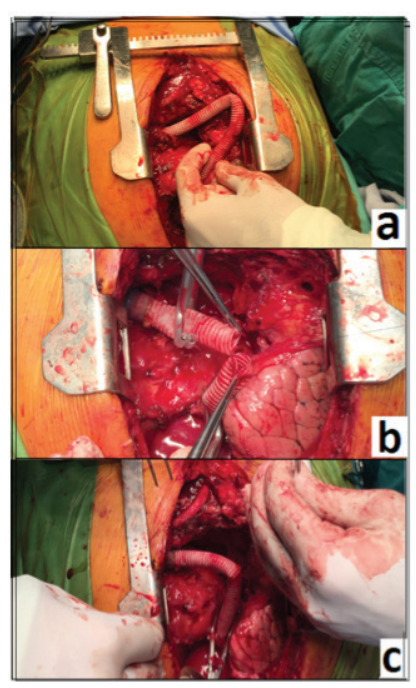

Figure 2. Too long outflow graft after adhesiolysis (A). Two clamps on graft and shortening by $6 \mathrm{~cm}$ (B). After 8 minutes (stop the LVAD pump) graft length was corrected (C).

the HVAD pump restarted at the initial pump settings. Heparin was neutralized using Protamine (Figure 2).

The postoperative period passed without complications. Holter monitoring showed sinus rhythm with rare episodes of VT without hemodynamic significance. TEE was done to better visualize the outflow graft and inflow cannula. Around the outflow graft a spout was observed with dimensions of 5.1 $\mathrm{cm}$ around the right atrium, $2.7 \mathrm{~cm}$ next to right ventriculum, with no compression on the actual graft, which was visualized along its length without kinking or angulation. MSCT indicated a small insignificant kinking (Figure 3).

At two months after hospital discharge, LVAD parameters were stable, with no LVAD alarms registered. Checkups after six, eight, and ten months showed regular pump parameters, general hemodynamic stability, and absence of VT and other arrhythmias.

\section{DISCUSSION}

Despite extended durability and improved long-term outcomes with LVAD use, there continues to be ongoing risk of complications, one of which is rhythm disturbances. Recent estimates suggest an incidence of 4.66 events per patient year. The occurrence of ventricular arrhythmias is found in $20-50 \%$ of LVAD-supported patients, with the greatest risk in the first month after device implantation [Griffin 2014].

Advanced cardiomyopathy impacts risk for arrhythmias at baseline in LVAD recipients. The insertion site of the inflow cannula has been correlated to morphological origins of monomorphic VT [Ziv 2005].

Both axial and centrifugal design continuous-flow LVADs provide constant unloading of the left ventricle throughout the entire cardiac cycle. Consequently, these devices are significantly more susceptible to preload and afterload changes [Vollkron 2007].

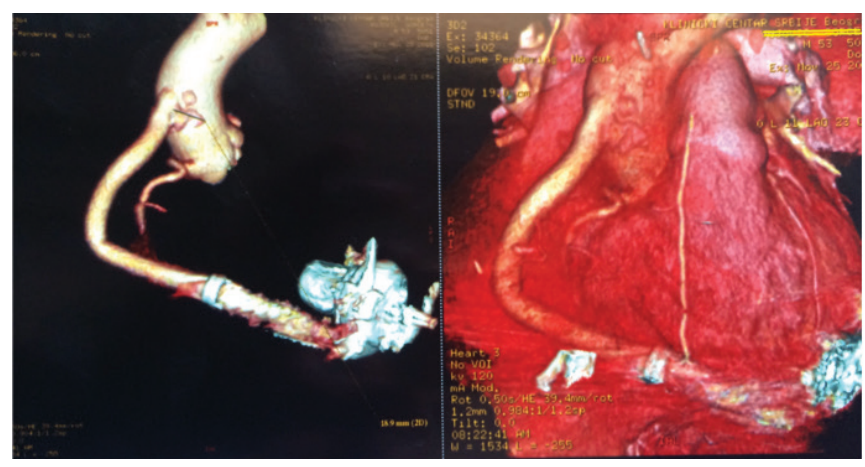

Figure 3. CT scan after correction and graft is visualized along its length, without kinking or angulation.

A study of LVAD suction events also showed a significant increase in monomorphic VT. Mismatch between LVAD outflow and inflow when left ventricular filling does not match LVAD output results in suction events. Consequently, the left ventricle is excessively unloaded and can partially or fully collapse, which can induce sustained or nonsustained ventricular arrhythmias that are monomorphic or polymorphic [Nakahara 2013].

Clinically relevant decreases in HVAD flow seem unlikely to be the result of outflow graft kinking. However, progressive angulation of the graft does result in increasing pressure gradients and decreased arterial perfusion pressures [Connellan 2013].

The presentation of ventricular arrhythmias in LVAD patients remains variable, with some patients able to tolerate ventricular arrhythmias with minimal symptoms. This is in part due to the LVAD's ability to maintain cardiac output independent of heart rate and atrioventricular synchrony. Nevertheless, impaired right ventricular filling due to ventricular arrhythmias can lead to inadequate LVAD flows, resulting in right heart failure, hemodynamic deterioration, ICD shocks, and even cardiac arrest in some patients. Uncontrollable ventricular arrhythmias and VT can be an indication for heart transplantation, bi-VAD, or total artificial heart; or, as in the present case, the need for reoperation tore-position the LVAD or its components [Nakahara 2013].

Our patient was identified as having obvious outflow graft kinking due to a too-long outflow graft. It was suspected that the outflow graft kinking caused changes in pressure gradients that were reflected in the myocardium, causing VT. Given that drug therapy did not give satisfactory response, it was decided that the patient would benefit from reoperation to shorten the outflow graft, despite increased surgical risk. Following reoperation there was no further VT, therefore, it appears that in some cases VT caused by kinking of a toolong outflow graft can benefit from reoperation.

\section{Conclusion}

Innovative strategies in VT prevention and improved clinical outcomes in patient populations with LVADs may be the result of better understanding of the characteristics that predispose these patients to VT. This case showed that excessive outflow graft length with considerable kinking was the culprit for new onset VT in a patient with multiple coughing 
episodes. Reoperation was necessary to correct the length of the graft, after which no further VT was noted.

\section{ACKNOWLEDGEMENTS}

The authors thank Kim Eleuteri, HeartWare, for editorial assistance. We thank the medical care staff of the medical team of Clinic for Cardiac Surgery Clinical Center of Serbia for their dedication in patient care and helping with the data collection.

\section{REFERENCES}

Connellan M, Muthiah K, Robson D, et al. 2013. Pressure and flow sequelae to kinking of the heartware HVAD outflow graft. J Heart Lung
Transplant 32:S280.

Griffin JM, Katz JN. 2014. The burden of ventricular arrhythmias following left ventricular assist device implantation. Arrhythm Electrophysiol Rev 3:145-8.

Nakahara S, Chien C, Gelow J, et al. 2013. Ventricular arrhythmias after left ventricular assist device. Circ Arrhythm Electrophysiol 6:648-54.

Vollkron M, Voitl P, Ta J, et al. 2007. Suction events during left ventricular support and ventricular arrhythmias. J Heart Lung Transplant 26:819-25

Ziv O, Dizon J, Thosani A, Naka Y, Magnano AR, Garan H. 2005. Effects of left ventricular assist device therapy on ventricular arrhythmias. J Am Coll Cardiol 45:1428-34. 